\title{
Simulation of Semi-Solid Material Mechanical Behavior Using a Combined Discrete/Finite Element Method
}

\begin{abstract}
M. SISTANINIA, A.B. PHILLION, J.-M. DREZET, and M. RAPPAZ
As a necessary step toward the quantitative prediction of hot tearing defects, a three-dimensional stress-strain simulation based on a combined finite element (FE)/discrete element method (DEM) has been developed that is capable of predicting the mechanical behavior of semisolid metallic alloys during solidification. The solidification model used for generating the initial solid-liquid structure is based on a Voronoi tessellation of randomly distributed nucleation centers and a solute diffusion model for each element of this tessellation. At a given fraction of solid, the deformation is then simulated with the solid grains being modeled using an elastoviscoplastic constitutive law, whereas the remaining liquid layers at grain boundaries are approximated by flexible connectors, each consisting of a spring element and a damper element acting in parallel. The model predictions have been validated against $\mathrm{Al}-\mathrm{Cu}$ alloy experimental data from the literature. The results show that a combined FE/DEM approach is able to express the overall mechanical behavior of semisolid alloys at the macroscale based on the morphology of the grain structure. For the first time, the localization of strain in the intergranular regions is taken into account. Thus, this approach constitutes an indispensible step towards the development of a comprehensive model of hot tearing.
\end{abstract}

DOI: $10.1007 / \mathrm{s} 11661-010-0491-0$

(C) The Minerals, Metals \& Materials Society and ASM International 2010

\section{INTRODUCTION}

HoT tearing is a spontaneous failure of semisolid metallic alloys that results in an intergranular fracture profile. This defect occurs during casting, near the end of solidification, especially in low-solute-content alloys. Hot tearing can be partially controlled by process changes such as mold design and/or cooling condition during solidification. However, a prerequisite to making such process changes efficiently is the development of a fundamental understanding of hot tearing formation and an ability to model its occurrence.

There are two main phenomena that lead to hot tearing: (1) a lack of liquid feeding at high fraction of solid $\left(g_{\mathrm{s}}\right)$ and (2) tensile or shear deformation transmitted through the partially coherent mushy zone. ${ }^{[1-7]}$ The formation of hot tears is similar to porosity formation in the sense that it is linked to a lack of liquid feeding in the mushy zone, but requires additionally shear or tensile deformation in order to separate, or pull apart, the solid network. These deformations occur because of the thermal gradients, solidification shrinkage, solid contraction, and mechanical constraints. Concurrently, the

M. SISTANINIA, Ph.D. Student, J.-M. DREZET, Senior Scientist, and M. RAPPAZ, Full Professor, are with the Computational Materials Laboratory, Ecole Polytechnique Fédérale de Lausanne, Lausanne 1015, Switzerland. Contact e-mail: meisam.sistaninia@ epfl.ch A.B. PHILLION, Assistant Professor, formerly with the Computational Materials Laboratory, Ecole Polytechnique Fédérale de Lausanne, is now with the School of Engineering, University of British Columbia, Kelowna V1V 1V7, Canada.

Manuscript submitted March 16, 2010.

Article published online October 19, 2010 distribution and amount of intergranular fluid control the feeding of localized regions within the mushy zone.

As grain morphology and size are characterized by a length scale much smaller than the process dimension, it is not surprising that most prior researchers have focused on macroscale averaging methods for modeling the interaction between a deforming solid skeleton and the intergranular fluid movement. ${ }^{[8-11]}$ In these works, a representative volume element (RVE) is assumed to contain a mixture of solid and liquid with local volume fractions $g_{\mathrm{s}}$ and $g_{l}$, respectively, interacting through the averaged conservation equations. ${ }^{[12]}$ Unfortunately, such approaches are unable to describe any strain inhomogeneity at the grain level, particularly at grain boundaries where hot tears form. Furthermore, crack initiation and propagation involve the creation of local discontinuities, which are difficult to consider when using an averaging approach.

In order to account for this granular nature of semisolids and strain inhomogeneity at the grain level, a number of so-called granular solidification models have been developed. Early models of this type considered a regular arrangement of grains that describe the ductility of the two-phase semisolid ${ }^{[13]}$ and liquid feeding. ${ }^{[14]}$ However, such regular arrangements of grains do not approximate the microstructure very well because all the solid grains percolate at the same time (i.e., when $g_{\mathrm{s}}$ reaches unity). Recently, Vernède and Rappaz $^{[15]}$ developed a two-dimensional (2D) solidification model proposed originally by Mathier et al., ${ }^{[16]}$ which does not have this shortcoming. In this model, grains are approximated by polyhedra based on the Voronoi diagram of a random set of nuclei, resulting in irregular grain arrangements. It has been shown that the 
solidification predictions of this model are close to those of more refined approaches such as the pseudofront tracking but at a much lower computational cost. ${ }^{[15]}$ The model is therefore ideally suited for granular simulations linking the behavior of a microscopic model to macroscopic properties of the material. Vernède et al. ${ }^{[17,18]}$ have used this 2D granular approach to simulate the fluid flow caused by grain movement and solidification shrinkage in an Al-Cu alloy. Phillion et al., ${ }^{[19,20]}$ using a similar approach based on 2D granular geometry, predicted the mechanical behavior of an equiaxed granular semisolid Al-Mg alloy.

Despite the novelty introduced by these granular models, it is clear that the behavior of the mushy zone cannot be modeled properly using 2D approaches because both semisolid deformation and fluid flow through a granular domain are inherently threedimensional (3D) problems. For example, although simultaneous continuity of both the solid and the liquid phases can exist in $3 \mathrm{D}$, a topological feature of the $2 \mathrm{D}$ geometry is that, for a given $g_{\mathrm{s}}$, only one of the two phases can be percolated through the domain.

In the present study, a 3D granular model based on the discrete element method (DEM) has been developed to predict the stress-strain behavior of a collection of equiaxed-granular grains in the semisolid state at a given $g_{\mathrm{s}}$. DEM is a numerical method for computing the motion and deformation of numerous particles. It was proposed in the 1970 s by Cundall and Strack ${ }^{[21]}$ for rock mechanics problems in which the continuity between the entities does not exist. It has proven to be useful for enhancing the understanding of granular and discontinuous materials in food science ${ }^{[22,23]}$ and mining applications. $^{[21,24]}$ Moreover, DEM is becoming widely accepted as an effective method for explaining experimentally observed facts in multiphase media. ${ }^{[25-30]} \mathrm{At}$ present, DEM has evolved from various disciplines including geomechanics, particle physics, and structural engineering.

The model presented in this work has been developed for a binary Al-2 wt pet $\mathrm{Cu}$ alloy because of its long freezing range, propensity for hot tear formation, and availability of experimental semisolid constitutive data. ${ }^{[8,10]}$ The mechanical behavior of the solid grains is modeled using a simple viscoplastic law, while the intergranular liquid has been replaced by connector elements. First, the methodology for generating the granular grain structure based on a solidification model is briefly described. ${ }^{[31]}$ Second, a description of the semisolid deformation model is provided with particular attention given to the link between the output of the solidification model and the input necessary for the mechanical calculation. The simulations are carried out using the finite element (FE) software Abaqus 6.8. ${ }^{[32]}$ Third, the deformation results from the FE/DEM simulation are discussed and validated against experimental data from the literature. ${ }^{[8,10]}$

\section{MODEL DEVELOPMENT}

\section{A. Generation of Discrete Elements Using a Solidification Model}

The geometry for a FE/DEM simulation of semisolid deformation is assumed to consist of equiaxed granular solid grains surrounded by liquid films or channels. The grains are meshed as solid elements. The liquid channels are not meshed but are replaced by connector elements. Generation of this geometry requires a methodology for simulating the solidification of numerous grains. Previous work by the authors ${ }^{[15-19,33]}$ has resulted in the development of a 3D granular solidification model known as GMS-3D, ${ }^{[31]}$ which is able to generate the required solid-liquid two-phase geometry at a fixed $g_{\mathrm{s}}$. In this model, the grain structure is derived from a Voronoi tessellation of random nucleation centers. After construction of a 3D Voronoi diagram, shown in Figure 1(a), each Voronoi region or grain (Figure 1(b)) is subdivided into polyhedral volume elements with the nucleation center as the summit and the Voronoi facet as the base (Figure 1(c)). These pyramids are divided further into tetrahedral elements to model solidification by subdividing each Voronoi facet into triangles (Figure 1(d)). As in the previous model designed for $2 \mathrm{D}$ geometries, ${ }^{[15-18]}$ the solute exchange between

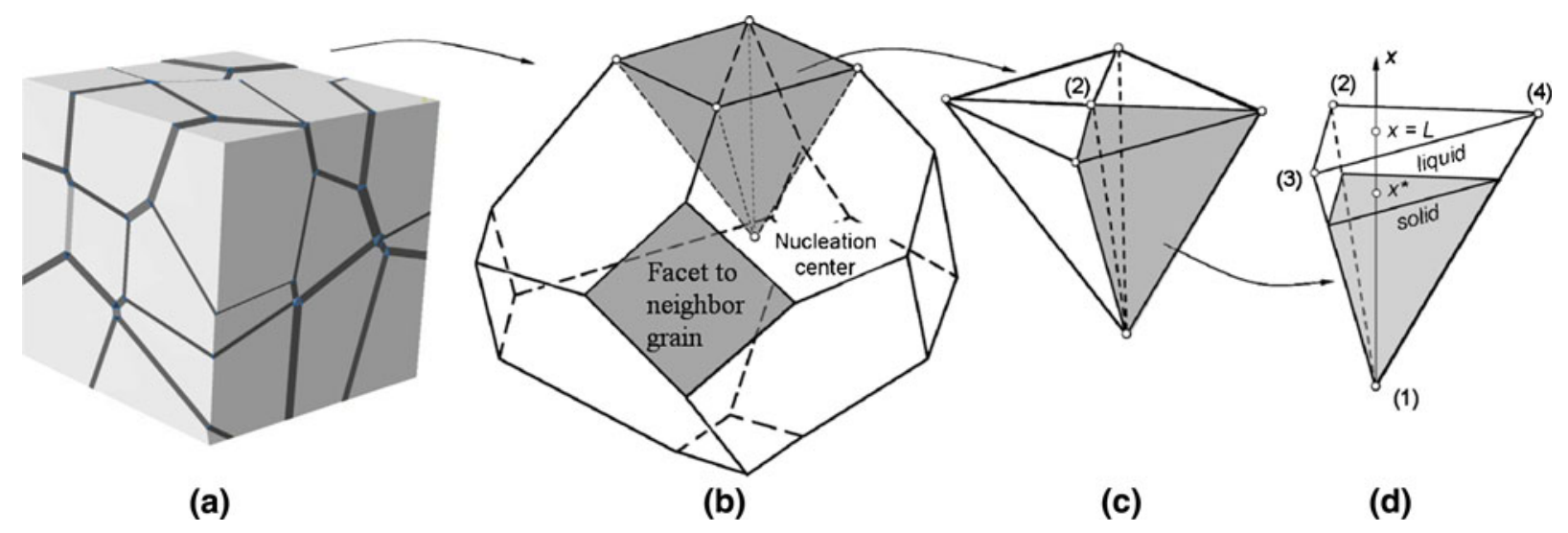

Fig. 1-A breakdown of the geometry describing the 3D granular solidification model, depicting (a) the entire model domain, (b) a polyhedral grain, $(c)$ a pentahedral volume element, and $(d)$ a single tetrahedral element showing both the solid portion (gray) and the liquid portion (clear). ${ }^{[31]}$ 
tetrahedral pyramids is neglected to reduce the microsegregation model to a $1 \mathrm{D}$ problem in spherical coordinates. Complete, or infinite, diffusion is assumed in the liquid, with some back-diffusion assumed in the solid. Thus, although all tetrahedral elements within a small pyramid solidify at the same speed because they have the same perpendicular length $L$ and hence the same Fourier number in the 1D solidification simulation, elements from different pyramids solidify at different rates (i.e., different Fourier numbers). It also should be noted that the facets of the solid polyhedral volume elements are not exactly continuous at edges. Such approximations are necessary to speed up the computation to predict solidification and percolation of a large number of grains.

As in the previous $2 \mathrm{D}$ solidification model, ${ }^{[15-18]}$ the master diffusion equation controlling the evolution of the solid-liquid interface in a tetrahedron can be derived from a solute balance integrated over the solid and liquid phases. This equation is given as follows ${ }^{[31]}$ :

$$
v^{*} x^{*^{2}}\left(k_{o}-1\right) C_{1}+\frac{1}{3}\left(L^{3}-x^{*^{3}}\right) \frac{\dot{T}}{m_{1}}+\left.x^{*^{2}} D_{\mathrm{s}} \frac{\partial C_{\mathrm{s}}}{\partial x}\right|_{x^{*}}=0[1]
$$

where $C_{\mathrm{s}}$ and $C_{1}$ are the solid and liquid composition, $v^{*}$ is the velocity of the interface, $x^{*}$ its actual position, $k_{o}$ is the partition coefficient, $D_{\mathrm{s}}$ is the diffusion coefficient in the solid, $\dot{T}$ is the cooling rate, and $m_{1}$ is the slope of the liquidus line. The evolution of the term $\left.\frac{\partial C_{s}}{\partial x^{*}}\right|_{x^{*}}$ is determined by solving the diffusion equation in the solid phase using a finite difference scheme with a Landau transformation to follow the interface, ${ }^{[34]}$ and the boundary conditions

$$
\left.\frac{\partial C_{\mathrm{s}}}{\partial x}\right|_{x=0}=0 \quad \text { and }\left.C_{\mathrm{s}}\right|_{x=x^{*}}=C_{\mathrm{s}}^{*}=k_{o} C_{1} .
$$

At the beginning of the solidification, the liquid channels are very wide, whereas near the end, they are narrow. Because small elements result in convergence issues during FE analysis, coalescence between two grains is assumed to occur when the thickness of the liquid channel between two elements is less than 0.4 pct of the average diameter of the grains. Although this hypothesis is not physically accurate, because the presence of thin liquid films of a few nanometers in size have been previously observed near the end of solidification, ${ }^{[35]}$ these small films are highly viscous and also tend to stick two surfaces together. Thus, for hot tearing, thin liquid channels act similar to solid-solid bonds.

As $g_{\mathrm{s}}$ increases, and the width of liquid channels is reduced, coalescence of two neighbor grains occurs (typically once $\left.g_{\mathrm{s}}>0.9\right) .{ }^{[36]}$ This behavior creates clusters of increasing size, which ultimately percolate throughout the domain (i.e., spread over the entire domain width). In Section III, it will be shown that the percolated solid at high $g_{\mathrm{s}}$ controls the mechanical resistance of the mushy zone.

\section{B. FEM Mechanical Calculation}

The output of the solidification calculation at a given value of $g_{\mathrm{s}}$ is a set of solid tetrahedral elements, with each one being in solid contact with three other solid elements belonging to the same grain. The fourth facet is either in solid contact with the symmetric element of the neighbor grain or is separated from it by a liquid channel if coalescence has not yet occurred at that location (Figure 1). This output is then translated into a finite element mesh using a $\mathrm{C}++$ subroutine within GMS-3D, which also automatically creates the Abaqus control file. As is shown in Figure 2, the following types of elements are involved in the finite element mesh of this geometry: (1) solid elements for the grains, (2) multipoint constraint (MPC) elements for the continuity between the facets of the same grain, (3) contact elements, and (4) flexible connector elements for the connectivity at grain boundaries. These last elements

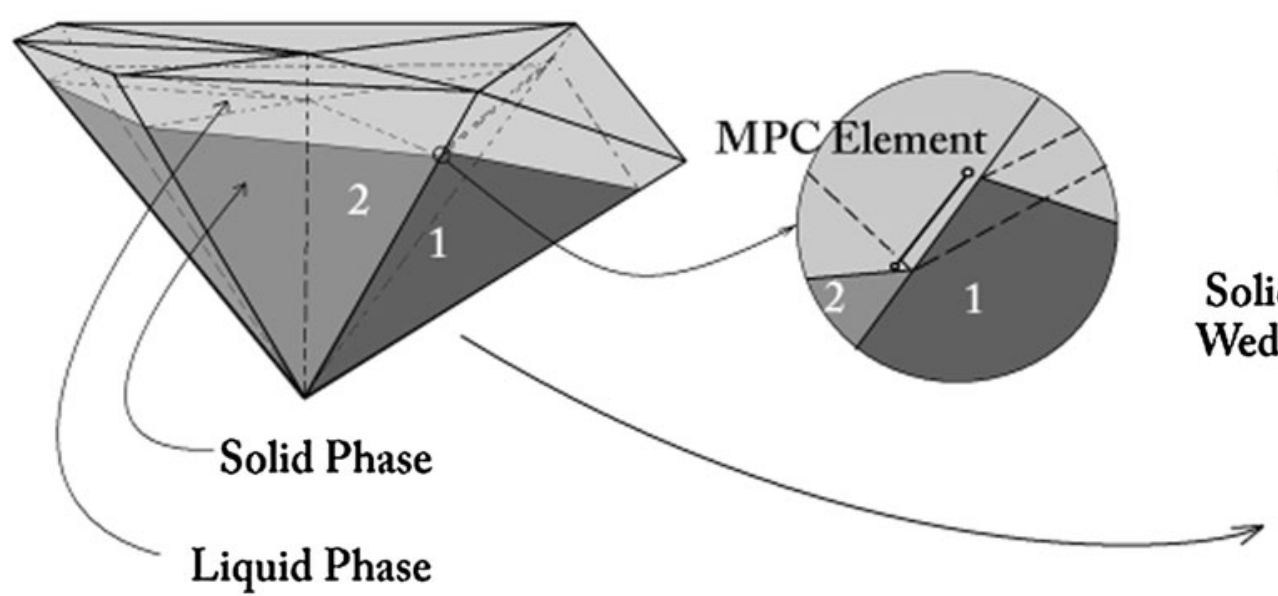

(a) (b)

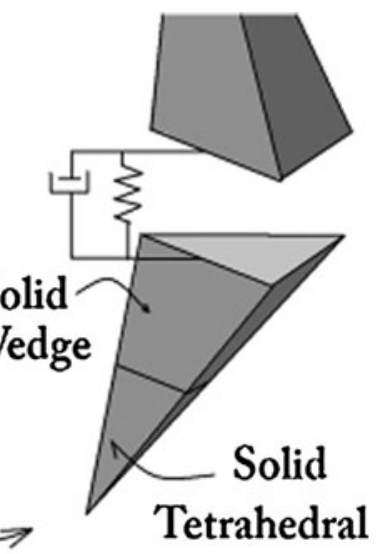

(c)

Fig. 2-The different elements involved in the FE model together with the connections between them, depicting ( $a$ ) one volume element (Fig. 1(c)) decomposed into a set of tetrahedrons, $(b)$ the MPC constraint between two nodes belonging to the same grain, and (c) a connector element between two nodes across a grain boundary. 
replace the liquid channels and are fitted to experimental data as explained below in more detail.

In the present simulations of semisolid deformation, the distribution of strain between the grains and in the intergranular region is of interest, whereas the detailed strain inhomogeneity within each grain is less relevant. Therefore, a coarse mesh for each grain has been used to simulate a 3D RVE that contains numerous grains.

\section{Solid elements}

Each solid tetrahedral element from the solidification calculation is split into two solid elements, a tetrahedron and a pentahedron, as shown in Figure 2(c). This split is made on a plane parallel to the solid-liquid interface or grain boundary. As a result, approximately 100 solid elements are present per grain for the FEM mechanical calculation. The mechanical behavior of these solid elements is assumed to be elastoviscoplastic. For the simulations at low $g_{\mathrm{s}}$ (i.e., $\left.g_{\mathrm{s}}<0.94\right)$, no solid bridges have formed between the grains, ${ }^{[36,37]}$ and the mechanical behavior of the free solid grains is relatively unimportant because most deformation is concentrated in the liquid films and because the stresses in the solid rarely exceed the yield stress. However, for simulations at high $g_{\mathrm{s}}$, the mechanical behavior of a domain containing numerous grain clusters is dictated increasingly by the behavior of the percolated grains. Physically, the mechanical behavior of each individual solid grain is anisotropic due to orientation dynamics (glide and climb) along preferred planes. Although such behavior is taken into account in detailed forming process models of solid materials, ${ }^{[38-40]}$ an idealized isotropic and elastoviscoplastic model is considered for the solid phase owing to the high number of randomly oriented grains in each cluster of the RVE. This simplified constitutive description has been used previously for examining the mechanical behavior of Al- $\mathrm{Cu}$ alloys. ${ }^{[5,8,41,42]}$ The isotropic elastic modulus $E=10 \mathrm{GPa}$ and isotropic Poisson coefficient $v_{\mathrm{s}}=0.30$ are taken from Reference 8 . As no hardening is observed in the experimental results of the $\mathrm{Al}-\mathrm{Cu}$ alloy at high temperature, ${ }^{[41,42]}$ the flow stress behavior of each grain beyond the elastic limit is assumed to be perfectly plastic and based on the following Norton-Hoff law:

$$
\sigma(\dot{\varepsilon})=k \cdot \dot{\varepsilon}^{m}
$$

where $\sigma$ is the stress, $\dot{\varepsilon}$ is the strain rate, $k$ is a flow stress coefficient, and $m$ is the strain-rate sensitivity coefficient of the material. Since the model is applied only over the range $0.9<g_{\mathrm{s}}<1$ and since the $m$ and $k$ do not change significantly over the corresponding range of temperature, ${ }^{[41]}$ constant values of $m$ and $k$ have been used. The values of $k=54.4 \mathrm{MPa}$ s and $m=0.195$ are taken from Reference 41. Please note that when $g_{\mathrm{s}}=1$, Eq. [2] must predict the mechanical behavior measured in a fully solid specimen.

\section{MPC elements}

As explained previously, each tetrahedron shown in Figure 2 solidifies independently from its neighbors within any given grain without exchanging solute. Thus, the continuity between the facets of each grain is not conserved along the solid-liquid interface. In order to conserve continuity within a grain, MPC elements are used to tie the facets of each grain to its own neighbors. The MPC element joining two nodes provides a universal connection between their degrees of freedom, ensuring that the displacement of one node is identical to that of its neighbor.

\section{Contact elements}

To prevent penetration between grains, contact elements are placed at the solid-liquid boundary of each grain. For this contact surface, a frictionless hard contact pressure-overclosure relationship is used. Thus, when surfaces are in contact, the pressure resulting from this interaction is transmitted, whereas the surfaces separate if the contact pressure reaches a value of zero. Computational cost is decreased using a finite-sliding, surface-to-surface contact formulation. ${ }^{[32]}$ In Abaqus, this formulation uses a path-based tracking algorithm that carefully considers the relative paths of points on the slave surface with respect to the master surface, within each increment, in order to determine the contact zone. $^{[32]}$

\section{Flexible connector elements}

The connectivity between two neighbor grains prior to coalescence is modeled using connector elements. As shown in Figure 2, each connector consists of one linkspring element and one axial damper element that act in parallel to approximate the effects of the intergranular liquid phase. The link-spring simulates the effect of the hydrostatic pressure within the liquid and thus controls the tensile forces required to separate two grains in a direction normal to the grain facets. This is because only the normal displacement leads to a volume change within the liquid and consequently to a change in hydrostatic pressure. The damper operates on the relative velocities of the grains, acting as a dashpot between the grains, which simulates the viscosity effects of the intergranular liquid and thus controls the shear forces between two grains.

The result of the link-spring and the axial damper replacing the liquid is a surface force vector between grains that contains the following components: a normal component $\left(t_{n}\right)$ and two shear tractions $\left(t_{s}, t_{e}\right)$. The nominal strain thus can be defined as follows:

$$
\varepsilon_{n}=\frac{u_{n}}{l_{\mathrm{o}}}, \quad \varepsilon_{s}=\frac{u_{s}}{l_{\mathrm{o}}}, \quad \varepsilon_{e}=\frac{u_{e}}{l_{\mathrm{o}}}
$$

where $u_{n}, u_{s}$, and $u_{e}$ are the three corresponding displacement components and $l_{\mathrm{o}}$ is the original thickness of the liquid channel between the two grains. Furthermore, the corresponding force exerted by the springdamper connector can be written as follows:

$$
\left(\begin{array}{c}
t_{n} \\
t_{s} \\
t_{e}
\end{array}\right)=\left(\begin{array}{ccc}
K & 0 & 0 \\
0 & 0 & 0 \\
0 & 0 & 0
\end{array}\right)\left(\begin{array}{c}
\varepsilon_{n} \\
\varepsilon_{s} \\
\varepsilon_{e}
\end{array}\right)+\frac{C}{A}\left(\begin{array}{c}
n \\
s \\
e
\end{array}\right) i
$$

where $(n, s, e)$ are the components of the unit vector along the damper, $i$ is the increasing rate of the length of the damper, $A$ is the area of the facet covered by each 
damper, $K$ is the elastic modulus of the spring, and $C$ is the damping coefficient of the damper. The damping coefficient can be thought of as a force per relative velocity. Although several types of elements in Abaqus allow the user to define a flexible joint, the cohesive element COH3D6 with nil shear modulus was used for the spring in this simulation, and the CONN3D2 element was used for the damper.

At present, constant values of $C$ and $K$ have been used, as the model is applied only over the range $0.9<g_{\mathrm{s}}<1$, which corresponds to the vulnerable range for hot tears formation and thus the interesting range for hot tearing simulations. The values of $C$ and $K$ were determined as follows. First, stress-strain curves obtained from a series of tensile deformation simulations were generated for a given $g_{\mathrm{s}}$, strain rate, and different values of $C$ and $K$. Second, these stress-strain curves were compared with semisolid tensile test experimental results. ${ }^{[8]}$ The values of $C$ and $K$ giving the best fit were determined and then used for all other simulation results. More details on this fitting procedure are provided in Section III. Although constant values of $C$ and $K$ have been used for the present work, it is clear that the liquid film resistance to separation and shear actually depends on its ability to be fed and thus on its location within the domain, as shown in 2D by Vernède et al. ${ }^{[17]}$ This type of feeding calculation is currently being developed and will be coupled iteratively, in a future work, to the present model so that the $C$ and $K$ values of each flexible connector can be varied during the simulation.

\section{Domain Size and Boundary Conditions}

As shown in Reference 43, the FE/DEM granular model requires a domain containing a minimum of 700 grains in order to accurately model semisolid deformation. Above this number, the behavior of the domain does not depend on the number and/or distribution of grains (i.e., the domain can be considered as a RVE of the mushy zone). In the simulations described below, the number of grains within the RVE is 864 , with an average grain size of $100 \mu \mathrm{m}$. Symmetry boundary conditions are imposed on the surfaces $x=0, y=0$, and $z=0$, while the surfaces $y=L_{y}$ and $z=L_{z}$ are free to move and the surface $x=L_{x}$ is connected to a reference node. The variables $L_{x}, L_{y}$, and $L_{z}$ refer to the length of the RVE along the $x, y$, and $z$ directions, respectively. To deform the RVE, an imposed displacement is applied to the reference node linked to the surface $x=L_{x}$, (i.e., a Dirichlet condition with fixed displacement is imposed on this boundary). The use of a reference node enables the bulk semisolid mechanical behavior to be obtained directly from the calculated force-displacement curve at this location.

\section{RESULTS AND DISCUSSION}

\section{A. Determination of $C$ and $K$}

The damping coefficient $C$ and spring constant $K$ for the flexible connectors are determined via a fitting

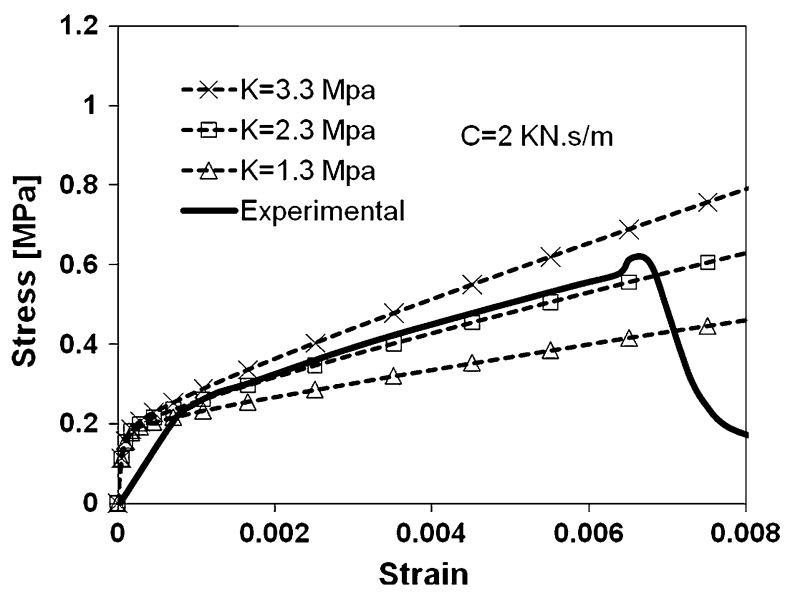

(a)

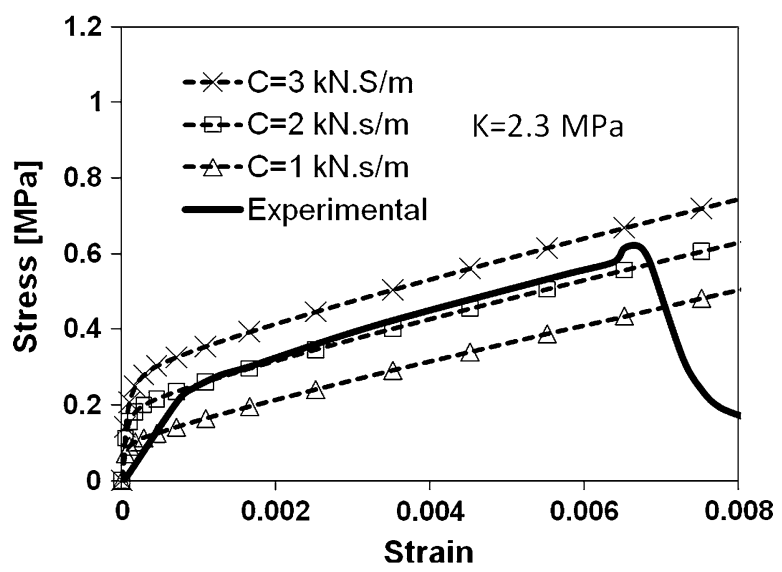

(b)

Fig. 3-A comparison between tensile test experimental results for $\mathrm{Al}-2 \mathrm{wt}$ pct $\mathrm{Cu}^{[8]}$ and the results of the model for $g_{\mathrm{s}}=0.94$ and for each $(a)$ spring moduli $\mathrm{K}$ and $(b)$ damping coefficients $\mathrm{C}$.

exercise and comparison with experimental data obtained for an $\mathrm{Al}-2$ wt pct $\mathrm{Cu}$ semisolid specimen. ${ }^{[8]}$ First, it must be noted that in order to deform the RVE at a uniform strain rate $\dot{\varepsilon}$, the velocity of the reference point is increased abruptly from zero to a uniform velocity given by $\left(\dot{\varepsilon} \times L_{x}\right)$. As the bulk stress of a semisolid material resulting from its viscosity (average "viscosity" of the solid and liquid) is a function of the velocity, the initial simulated stress-strain behavior presents a sharp stress increase. A similar initial stress increase is also actually observed in experimentally measured semisolid tensile stress-strain curves. ${ }^{[8,44]}$ According to this initial increase, the damping coefficient $C$ can be estimated, while $K$ can be estimated from the slope of the stress-strain curve after this initial transient.

The fitting exercise is shown in Figure 3, in which the simulated bulk stress-strain curves obtained at $g_{\mathrm{s}}=0.94$ and $\dot{\varepsilon}=0.001 \mathrm{~s}^{-1}$ for various values of $K$ (Figure 3(a)) and $C$ (Figure 3(b)) are compared with tensile test experimental results at the same $g_{\mathrm{s}} \cdot{ }^{[8]}$ In this figure, the experimental curve is shown as a solid line, while the simulated curves are shown as dashed lines with symbols. As is shown when comparing the curves of 
Figures 3(a) and 3(b), the initial stress increase is a function of $C$ but not of $K$. However, the slope of these curves after the primary increase depends only on $K$ but not on $C$. Using this series of simulations, a value of $C=2 \times 10^{3} \mathrm{~N} \mathrm{~s} / \mathrm{m}$ for the damping coefficient and a value of $K=2.3 \mathrm{MPa}$ for the elastic modulus of the spring were chosen. These values will be used in all simulation results presented hereafter. Note also that, since there is no failure model included in the numerical computations, the stress-strain curves do not exhibit the maximum observed in the experimental data.

\section{B. Tensile Deformation}

In Figure 4, a comparison is made between the simulated stress-strain predictions and the experimental results for the same $\mathrm{Al}-2 \mathrm{wt}$ pet $\mathrm{Cu}$ alloy from ${ }^{[8]}$ over a range of fractions of solid between 0.92 and 0.98 at a strain rate of $\dot{\varepsilon}=0.001 \mathrm{~s}^{-1}$. Beginning with the experimental data, it is shown that, for all tests, the stress increases with increasing strain as expected and reaches a maximum value $\sigma_{\max }$ before failure occurs. However, two different types of behavior are observed. For $g_{\mathrm{s}}=0.92,0.94$, and 0.96 , the stress gradually increases until it reaches the maximum value $\sigma_{\max }$, whereas at $g_{\mathrm{s}}=0.98$, the stress increase is larger because more grains have percolated, thus increasing the stiffness of the semisolid material. Some hardening is also visible in all four curves. During the initial stages of deformation, the role of the percolated solid is dominant, but once the percolated solid yields, the behavior is dictated mainly by the contact and deformation of the free grains. Hence, although the behavior of the solid grains is actually elastic-perfectly plastic, the behavior of the entire semisolid material is not, as is shown in Figure 4. Instead, it undergoes geometric strain hardening ${ }^{[19]}$ during deformation. An initial stress increase can also be observed in the results for $g_{\mathrm{s}}=0.94$ and $g_{\mathrm{s}}=0.98$. In the same figure, the results of the FE/DEM model are also presented. As is shown, they correctly reproduce

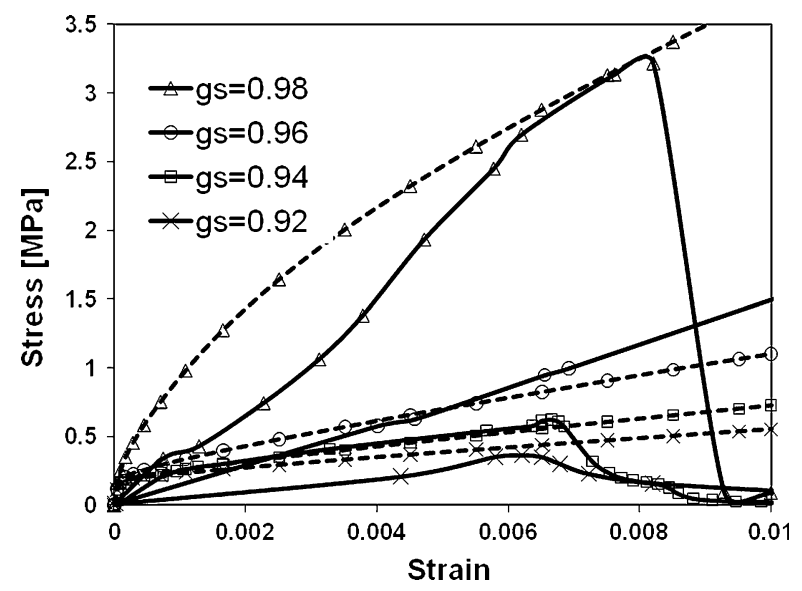

Fig. 4-A comparison between the tensile experimental results of partially solidified $\mathrm{Al}-2$ wt pet $\mathrm{Cu}$ alloys ${ }^{[8]}$ (continuous curves) and simulation results (dashed line curves) for the following fractions of solid: $\times g_{\mathrm{s}}=0.92\left(T=883 \mathrm{~K}\left(610^{\circ} \mathrm{C}\right)\right) ; \square g_{\mathrm{s}}=0.94 ; \bigcirc g_{\mathrm{s}}=0.96$ $\left(T=858 \mathrm{~K}\left(585^{\circ} \mathrm{C}\right)\right) ; \Delta g_{\mathrm{s}}=0.98\left(T=824 \mathrm{~K}\left(551^{\circ} \mathrm{C}\right)\right)$. the general trends of the experimental curves for the four values of $g_{\mathrm{s}}$, with $g_{\mathrm{s}}=0.94$ showing the best agreement since it was used in Figure 3 for the calibration of $C$ and $K$.

To investigate the tensile mechanical behavior of the model at different strain rates, the simulated results are compared with experimental results of Reference 10, which also used an Al-2 wt pet $\mathrm{Cu}$ alloy. As is shown in Figure 5, the results of the model accurately reproduce the general trends of the experimental curves obtained at 0.001 and $0.004 \mathrm{~s}^{-1}$ strain rates. The values for $C$ and $K$ were identical in both cases and were taken from Section III-A. The results from two other simulations, using strain rates of $10^{-4}$ and $10^{-2} \mathrm{~s}^{-1}$, also are shown. Although these results could have been produced using an averaging constitutive model (e.g., The one by Mathier containing an internal variable related to the evolution of coherency) $)^{[10]}$ containing an internal variable related to the evolution of coherency, such alternative approaches cannot give access to stress and strain inhomogeneities such as those shown in Figures 6 and 7.

Figure 6 shows strain contours from cross sections within the RVE for three values of $g_{\mathrm{s}}\left(g_{\mathrm{s}}=0.92,0.96\right.$, and 0.98 ), outlined in Figure 4, when the overall or bulk strain is 1 pct. The deformation is horizontal and to the right. As is shown, strain localization is concentrated within the liquid films located between the grains (i.e., is accommodated by the dampers and springs). For the two images at lower $g_{\mathrm{s}}$, Figures 6(a) and (b), the strain is fairly well distributed in the liquid channels perpendicular to the tension direction. However, when $g_{\mathrm{s}}=0.98$ (Figure 6(c)), the strain appears to be localized along few preferential paths. Also, the maximum local strain for $g_{\mathrm{s}}=0.98$ is predicted to be about four times larger than the maximum local strain for $g_{\mathrm{s}}=0.92$ and is two orders of magnitude larger than the bulk strain of the RVE.

Figure 7 shows the contour plots of the Von Mises stress in (MPa) for $g_{\mathrm{s}}=0.98$ at (1) the boundary surface and (2) a slice inside the RVE. In this figure, it is shown

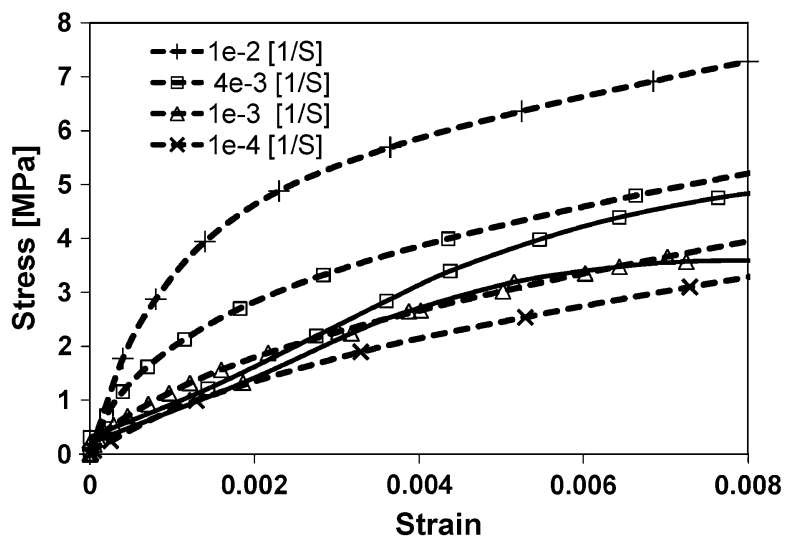

Fig. 5-A comparison between the tensile experimental results of partially solidified $\mathrm{Al}-2$ wt pet $\mathrm{Cu}$ alloys ${ }^{[10]}$ (continuous curves) and simulation results (dashed line curves) for $g_{\mathrm{s}}=0.99(T=813 \mathrm{~K}$ $\left.\left(540{ }^{\circ} \mathrm{C}\right)\right)$ and different strain rates. 


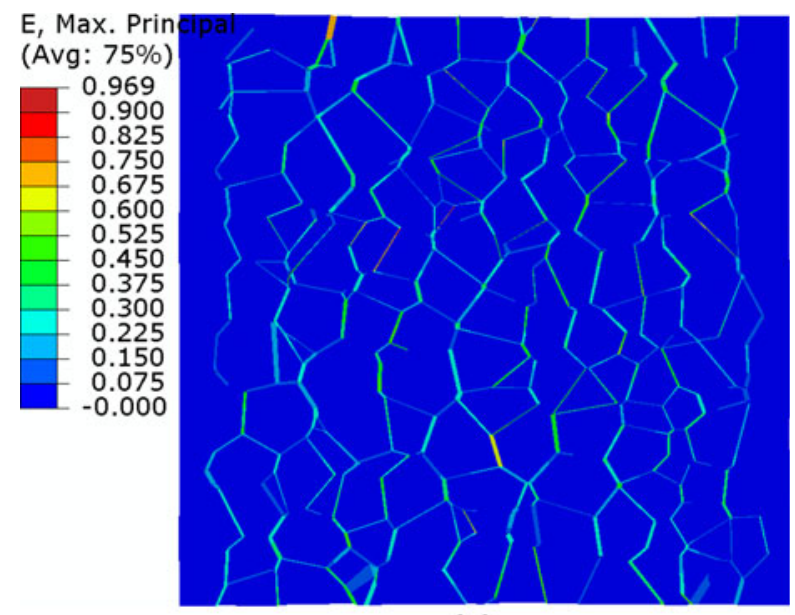

(a)

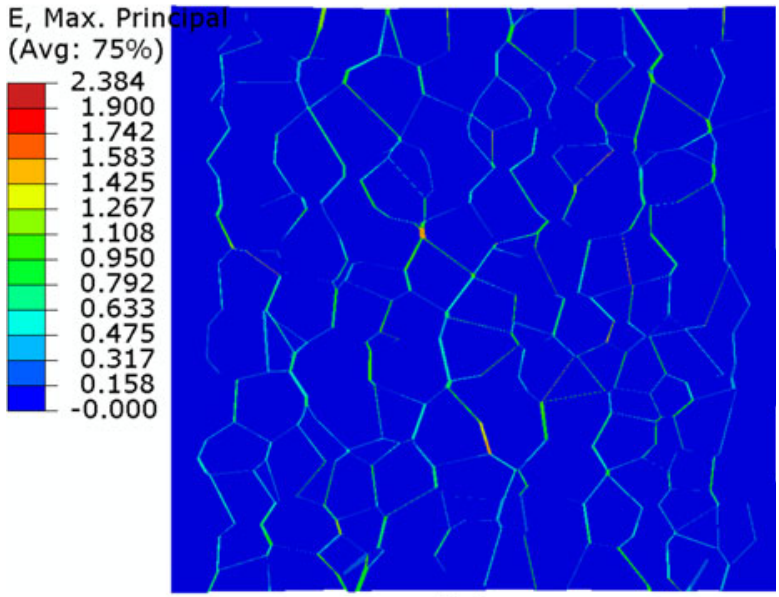

(b)

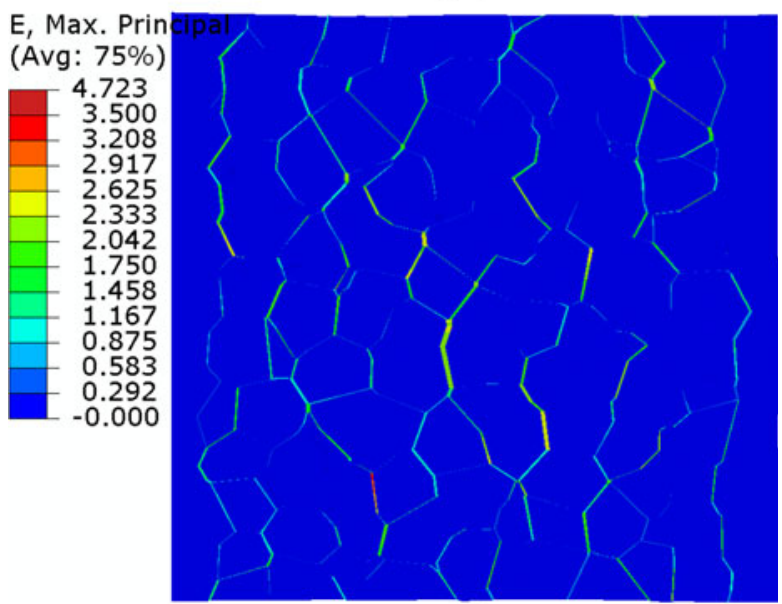

(c)

Fig. 6-Finite-element simulation showing strain localization through an isothermal mushy zone volume element $(1.2 \times 1.2 \times$ $6 \mathrm{~mm}^{3}$ ) under tensile deformation for $(a) g_{\mathrm{s}}=0.92$, (b) $g_{\mathrm{s}}=0.96$, and $(c) g_{\mathrm{s}}=0.98$.

that percolated grains transmit the load from the left to the right face of the RVE. In this case, solid bridges act as the main obstacles to the fracture of the semisolid material in tension.

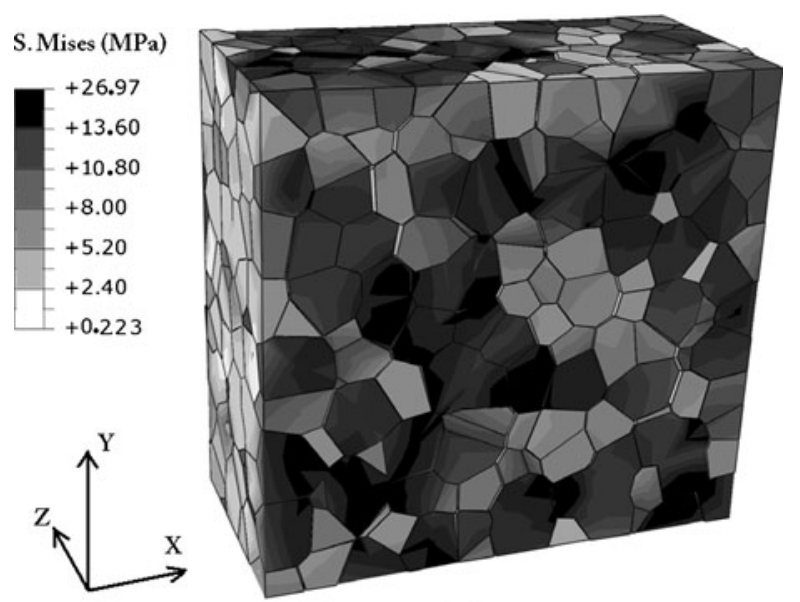

(a)

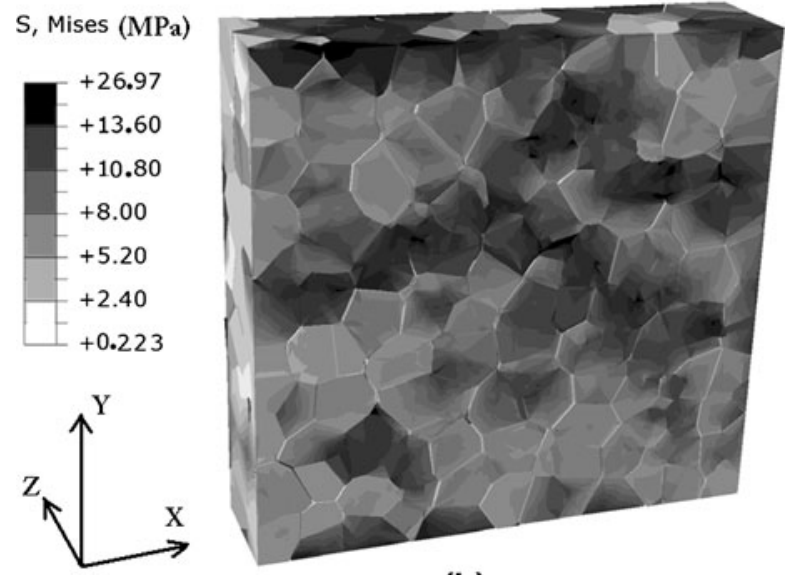

(b)

Fig. 7-Contour plot of the Von Mises stress in $\mathrm{MPa}$ for tensile deformation at $g_{\mathrm{s}}=0.98$ on $(a)$ outer surface $(b)$ a cross section inside the domain.

\section{Compression Deformation}

It is well known that the tensile and compressive behavior of semisolid materials are drastically different. ${ }^{[8,45,46]}$ In the mushy zone, compressive deformation is similar to the squeezing of a sponge, causing the intergranular liquid to flow out and the pressure within this liquid to increase. Figure 8 shows the simulated mechanical behavior of the mushy zone in compression as a function of $g_{\mathrm{s}}$ for the same parameters $C$ and $K$ used in tension. Although at low strain, the behavior of the semisolid in tension and compression is similar, the compressibility of the mush decreases and approaches the incompressibility of a fully solid structure $\left(g_{\mathrm{s}}=1\right)$ as soon as the liquid between the grains has been removed and the grains come into contact with each other. The start of incompressibility is defined as the inflection point in a stress-strain curve (i.e., $d^{2} \sigma / d \varepsilon^{2}=0$ ) and is the point in the model when the contact elements activate at the grain boundaries. As is shown by the large black circles in Figure 8, the start of incompressibility occurs at $\varepsilon=0.003$ for $g_{\mathrm{s}}=0.98$ but increases to $\varepsilon=0.011$ and then $\varepsilon=0.016$ when $g_{\mathrm{s}}$ decreases to 0.96 


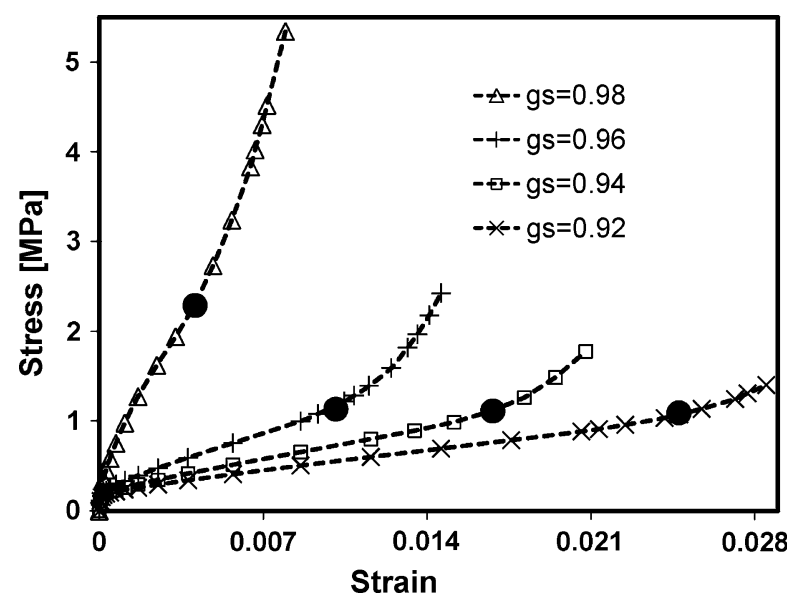

Fig. 8-Compression behavior of $\mathrm{Al}-2$ wt pct $\mathrm{Cu}$ alloys. Stress vs strain curves from the model for the following fractions of solid: $\times g_{\mathrm{s}}=0.92 ; \square g_{\mathrm{s}}=0.94 ;+g_{\mathrm{s}}=0.96 ; \Delta g_{\mathrm{s}}=0.98$. The large black circles represent the beginning of incompressibility.

and 0.94 , respectively, and more liquid is present between grains. However, please note that the maximum stress that can be modeled is limited because of contactconvergence difficulties.

\section{Shear Deformation}

Figure 9 shows the simulated mechanical behavior of the RVE under shear deformation and the experimental results of an $\mathrm{Al}-2 \mathrm{wt}$ pct $\mathrm{Cu}$ alloy being sheared in the semisolid state between two coaxial cylinders. ${ }^{[8]}$ The experimental test consists of imposing a constant velocity to the inner cylinder along the longitudinal axis while the experimental test specimen is held at a given temperature. As the spacing between the two cylinders remains constant, this test did not correspond to a simple shear but rather to an in-plane shear with a small tensile component along the cylinder radius. Although this component is small at low strain, it becomes important and leads to higher values of the Von Mises stress with increasing strain.

As is shown in Figure 9, the agreement between the simulated shear deformation curves and the experimental results is satisfactory for low strain and low $g_{\mathrm{s}}$ ( $g_{\mathrm{s}}=0.92$, i.e., prior to the formation of solid bridges). At higher strain, however, the calculated stress for $g_{\mathrm{s}}=0.92$ is below the experimental curve. This result can probably be attributed to the difference between the simulated simple shear and the experimental inplane shear. At high $g_{\mathrm{s}}\left(g_{\mathrm{s}}=0.98\right)$, the model also reproduces correctly the initial behavior of the mushy zone but then overpredicts the stress beyond approximately $\varepsilon=0.3$. This deviation can be linked to the rupture of the connections (bridges) between percolated grains. Although thin films of liquid do not allow two grains to be pulled apart in tension without liquid feeding, these films do allow grains to slip across each other because no volume change is involved. As mentioned, this model assumes the thin liquid channels to act similar to solid-solid bonds. To reproduce the

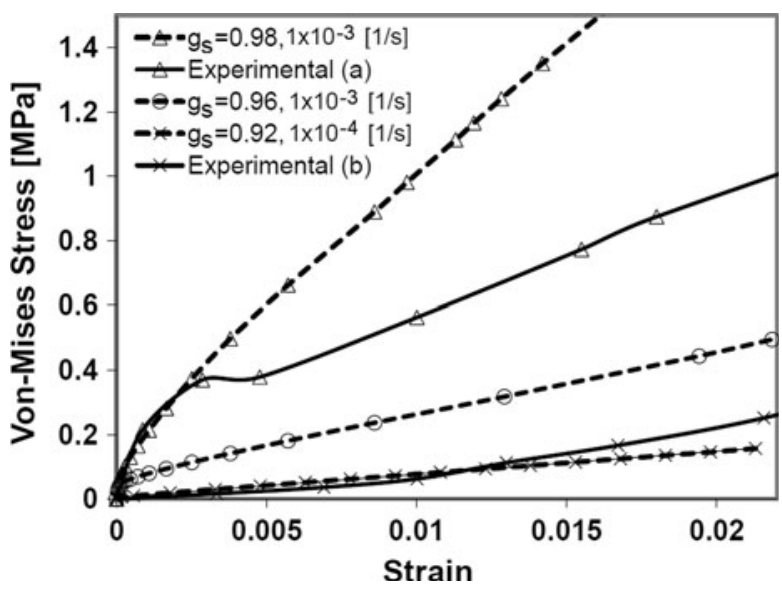

Fig. 9-Shear behavior of Al-2 wt pet $\mathrm{Cu}$ alloys. Stress vs strain curves from experimental data (continuous curves) $(a) \mathrm{T}=823 \mathrm{~K}$ $\left(550{ }^{\circ} \mathrm{C}\right), \dot{\varepsilon}=1 \times 10^{-3} \mathrm{~s}^{-1}$ and $(b) \quad \mathrm{T}=883 \mathrm{~K} \quad\left(610^{\circ} \mathrm{C}\right), \quad \dot{\varepsilon}=1 \times$ $10^{-4} \mathrm{~s}^{-1[8]}$ and from the model (dashed line curves) for the following fractions of solid: $\times g_{\mathrm{s}}=0.92 ; \bigcirc g_{\mathrm{s}}=0.96$; and $\Delta g_{\mathrm{s}}=0.98$.

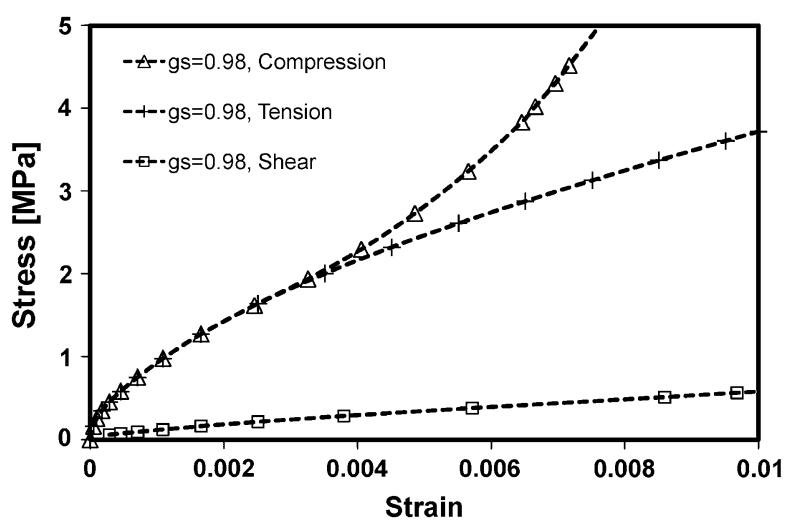

Fig. 10-A comparison of the mechanical behavior of the mushy zone under shear, compressive, and tensile loading at $g_{\mathrm{s}}=0.98$.

experimentally observed behavior of the mushy zone at high $g_{\mathrm{s}}$ under shear deformation, a failure criterion of the solid-solid bonds in shear would need to be added to the model.

\section{E. Comparison of the Three Deformation Modes}

The simulation results presented for tension, compression, and shear show that the model can predict different behavior for the different mechanical tests. Figure 10 shows a comparison of the behavior of the mushy zone under these three deformation modes. As is shown, the deformability (i.e., the amount of strain for a given stress) is lower in compression as compared with tension because the intergranular liquid can flow out to allow for solid contact between grains. The inflection point and/or the beginning of incompressibility is the starting point of the deviation of the compressive curve from the tensile curve. Below this point, the mechanical behavior of the mushy zone is controlled by the deformation of the percolated grains and the connector 
elements and thus is the same. However, above the inflection point, the mechanical behavior in compression is affected by the solid contacts between the grains, whereas in tension, the behavior remains controlled only by the deformation of the percolated grains and the connector elements.

Under shear deformation, the normal link-spring behaves in a similar fashion to intergranular liquid and allows the solid grains to slip over each other. In this case, the semisolid deformability is higher as compared with the tensile behavior. The experimental results for aluminum alloys also demonstrate this variability and show different semisolid tension, compression, and shear mechanical behaviors.

\section{CONCLUSIONS}

A 3D granular model has been developed based on discrete elements to predict the mechanical behavior of semisolid metallic alloys. The initial geometry of the semisolid at a given volume fraction of solid was obtained from a 3D granular model of solidification. ${ }^{[31]}$ The elements making up the solid grains were modeled using elastoviscoplastic behavior, along with contact surfaces to limit intergrain penetration. The grain interactions prior to complete solidification (i.e., the behavior of the intergranular liquid) were simulated through the use of connector elements. Each connector consisted of one link-spring element and one damper element acting in parallel to approximate the hydrostatic and shear behavior of the liquid. The model predictions have been validated successfully against prior literature experimental results, ${ }^{[8,10]}$ using only two fixed parameters. Both parameters $C$ and $K$ were held constant over the range of $g_{\mathrm{s}}$ investigated.

In comparison with averaging methods using internal variables and/or complex behavior, the combined $\mathrm{FE} /$ DEM approach can predict the inhomogeneous strain distribution in a semisolid RVE. This technique can be used because the interest with respect to hot tearing and other solidification defects lies in understanding the mechanical behavior of a collection of grains with a random distribution of nucleation centers, whereas the detailed deformation within a single grain and/or the fluid velocity within a single channel can be approximated. The limitations of this model are as follows:

1. The model can predict the behavior of the mushy zone only in the range of $g_{\mathrm{s}}$ between 0.9 and 1 . For $g_{\text {s }}$ lower than 0.9 , the mechanical properties of the solid grains and the values used for $C$ and $K$ must be reevaluated.

2. The model can predict the behavior of the mushy zone only under small deformations and limited strain rate.

3. The model cannot predict the behavior of the mushy zone after damage initiation because such prediction requires the consideration of the intergranular liquid flow induced by solidification shrinkage and by the opening of wet grain boundaries. This limitation is the subject of current research by the authors to localize the pressure drop in the remaining liquid and to propose a defect nucleation criterion.

\section{ACKNOWLEDGMENTS}

The authors would like to thank the Swiss Competence Centre for Materials Science and Technology (CCMX) and partner companies within the thematic area "Multi-scale, multi-phenomena modelling of metallic systems" for funding this research.

\section{REFERENCES}

1. J. Campbell: Mater. Sci. Technol., 1991, vol. 7, pp. 885-94.

2. T.W. Clyne and G.J. Davies: ICME, 1975, vol. 68, pp. 238-44.

3. T.W. Clyne, M. Wolf, and W. Kurz: Metall. Trans. B, 1982, vol. 13B, pp. 259-66.

4. J.A. Dantzig and M. Rappaz, eds.: Solidification, EPFL Press, Lausanne, Switzerland, 2009.

5. J.M. Drezet, O. Ludwig, M. M'Hamdi, H.G. Fjaer, and C.L. Martin: Light Metals, TMS, Warrendale, PA, 2004, pp. 655-60.

6. C.A. Monroe, C. Beckermann, and J. Klinkhammer: in Modeling of Casting, Welding, and Advanced Solidification Processes-Xii, S.L. Cockcroft and D.M. Maijer, eds., TMS, New York, NY, 2009

7. M. Rappaz, J.M. Drezet, and M. Gremaud: Metall. Mater. Trans. A, 1999, vol. 30A, pp. 449-55.

8. O. Ludwig, J.M. Drezet, C. Martin, and M. Suéry: Metall. Mater. Trans. A, 2005, vol. 36A, pp. 1525-35.

9. V. Mathier, J.M. Drezet, and M. Rappaz: Model Simul. Mater. Sci. Eng., 2007, vol. 15, pp. 121-34.

10. V. Mathier, S. Vernède, P. Jarry, and M. Rappaz: Metall. Mater. Trans. A, 2009, vol. 40A, pp. 943-57.

11. M. M'Hamdi, A. Mo, and C.L. Martin: Metall. Mater. Trans. A, 2002, vol. 33A, pp. 2081-93.

12. J. Ni and C. Beckermann: Metall. Trans. B, 1991, vol. 22B, pp. 349-61.

13. D.J. Lahaie and M. Bouchard: Metall. Mater. Trans. A, 2001, vol. 32A, pp. 697-705.

14. W.O. Dijkstra, C. Vuik, A.J. Dammers, and L. Katgerman: in Solid Proc \& Microst: Symp Hon, W. Kurz, M. Rappaz, C. Beckerman, and R. Trivedi, eds., TMS, New York, NY, 2004, pp. 151-56.

15. S. Vernède and M. Rappaz: Acta Mater., 2007, vol. 55, pp. 170310 .

16. V. Mathier, A. Jacot, and M. Rappaz: Model Simul. Mater. Sci. Eng., 2004, vol. 12, pp. 479-90.

17. S. Vernède, J.A. Dantzig, and M. Rappaz: Acta Mater., 2009, vol. 57, pp. 1554-69.

18. S. Vernède, P. Jarry, and M. Rappaz: Acta Mater., 2006, vol. 54, pp. 4023-34

19. A.B. Phillion, S.L. Cockcroft, and P.D. Lee: Acta Mater., 2008, vol. 56, pp. 4328-38.

20. A.B. Phillion, S.L. Cockcroft, and P.D. Lee: Model Simul. Mater. Sci. Eng., 2009, vol. 17, pp. 366-75.

21. P.A. Cundall and O.D.L. Strack: Geotechnique, 1979, vol. 29, pp. 47-65.

22. Y.J. Sun and W.L. Xu: 15th Int. Conf. on Mechatronics and Machine Vision in Practice (M2vip), Auckland, New Zealand, 2008, pp. 201-07.

23. K.M. Wright, J. Sprunt, A.C. Smith, and B.P. Hills: Int. J. Food Sci. Tech., 2003, vol. 38, pp. 351-60.

24. P.A. Cundall: Proc. of the Institution of Civil Engineers-Geotechnical Engineering, 2001, vol. 149, pp. 41-47.

25. M.A. Delele, E. Tijskens, Y.T. Atalay, Q.T. Ho, H. Ramon, B.M. Nicolai, and P. Verboven: J. Food Eng., 2008, vol. 89, pp. 33-41.

26. G. Frenning: Comput. Meth. Appl. Mech. Eng., 2008, vol. 197, pp. $4266-72$. 
27. K. Han, D. Peric, A.J.L. Crook, and D.R.J. Owen: Eng. Computation, 2000, vol. 17, pp. 593-619.

28. A. Munjiza and K.R.F. Andrews: Int. J. Num. Meth. Eng., 2000, vol. 49 , pp. 1377-96.

29. H. Peron, J.Y. Delenne, L. Laloui, and M.S. El Youssoufi: Computers Geotechnics, 2009, vol. 36, pp. 61-69.

30. T.G. Sitharam: Curr. Sci., 2000, vol. 78, pp. 876-86.

31. A.B. Phillion, J.L. Desbiolles, and M. Rappaz: in Modeling of Casting, Welding, and Advanced Solidification Processes-Xii, Vancouver, Canada, S. Cockcroft and D. Maijer, eds., TMS, Warrendale, PA, 2009, pp. 353-60.

32. Abaqus: Abaqus Theory Manual Version 6.8-2, RI 02909-2499, 2008.

33. A.B. Phillion, S. Vernede, M. Rappaz, S.L. Cockcroft, and P.D. Lee: Int. J. Cast Met. Res., 2009, vol. 22, pp. 240-43.

34. V.R. Voller and S. Sundarraj: Mater. Sci. Technol., 1993, vol. 9, pp. $474-81$

35. I. Farup, J.M. Drezet, and M. Rappaz: Acta Mater., 2001, vol. 49, pp. 1261-69.

36. M. Rappaz, A. Jacot, and W.J. Boettinger: Metall. Mater. Trans. $A$, 2003, vol. 34A, pp. 467-79.

37. M. Rappaz, J.M. Drezet, P.D. Grasso, and A. Jacot: in Modeling of Casting, Welding and Advanced Solidification Processes- $X$,
D. Stefanescu, J. Waren, M. Jolly, and M. Krane, eds., TMS, Warrendale, PA, 2003, pp. 53-60.

38. P. Van Houtte, A. Van Bael, and S. He: Proc. Of the 9th Int. Conf. on Numerical Methods in Industrial Forming Processes, 2007, pp. 159-64.

39. P. Van Houtte, A. Van Bael, and M. Seefeldt: 5th Int. Conf. on Processing \& Manufacturing of Advanced Materials, Vancouver, Canada, 2006, pp. 3454-59.

40. P. Van Houtte, A. Van Bael, and M. Seefeldt: Fund. Deform. Anneal., 2007, vol. 550, pp. 13-22.

41. B. Magnin, L. Maenner, L. Katgerman, and S. Engler: Mater. Sci. Forum, 1996, vols. 217-222, pp. 1209-14.

42. P. Wisniewski and H.D. Brody: in Modeling of Casting, Welding, and Advanced Solidification Processes, M. Rappaz, M.R. Ozgu, and K.W. Mahin, eds., TMS, Warrendale, PA, 1991, pp. 273-78.

43. M. Sistaninia, A.B. Phillion, J.M. Drezet, and M. Rappaz: 49th Annual Conf. of Metallurgists, Vancouver, Canada, 2010.

44. S. Vernède: Ph.D. Dissertation, Ecole Polytechnique Federale de Lausanne, Lausanne, Switzerland, 2007.

45. D.G. Eskin and S.L. Katgerman: Prog. Mater. Sci., 2004, vol. 49, pp. 629-711.

46. W.M. van Haaften, W.H. Kool, and L. Katgerman: Mater. Sci. Eng. A, 2002, vol. 336, pp. 1-6. 